\title{
ERRATUM
}

\section{Systemic lupus erythematosus (SLE) and chromosome 16: confirmation of linkage to 16q12-13 and evidence for genetic heterogeneity}

Swapan K Nath, Bahram Namjou, David Hutchings, C Phillip Garriott, Catherine Pongratz, Joel Guthridge and Judith A James

European Journal of Human Genetics (2004) 12, 688. doi:10.1038/sj.ejhg.5201253

Correction to: European Journal of Human Genetics (2004); doi:10.1038/sj.ejhg.5201209; Published online: 28 April 2004.
The last authors name was published incorrectly, the correct version is shown above. 